\title{
Microencapsulation of Gac (Momordica cochinchinensis Spreng.) fruit oil in Ca-Alginate bead produced through Jetcutter
}

\author{
Vinh Truong*, Phuong N. M. Ta, \& Phuong T. Nguyen
}

Department of Chemical Engineering and Processing, Nong Lam University, Ho Chi Minh City, Vietnam

\author{
ARTICLE INFO \\ Research Paper \\ Received: June 07, 2020 \\ Revised: July 22, 2020 \\ Accepted: August 14, 2020 \\ Keywords \\ Alginate \\ Gac fruit oil \\ Jetcutter \\ Microencapsulation \\ Momordica cochinchinensis Spreng. \\ * Corresponding author \\ Truong Vinh \\ Email: tv@hcmuaf.edu.vn
}

\begin{abstract}
The Gac (Momordica cochinchinensis Spreng.) fruit oil was microencapsulated in Ca-Alginate bead by Jetcutter equipment. Experiments showed that the effects of emulsion preparation and equipment parameters were significant on particle size, microencapsulation yield, loading capacity and solids recovery efficiency of the device. At alginate concentration of $2.5 \%$, the bead had a spherical size of $1.07-1.18 \mathrm{~mm}$ when the fluid flow was $14 \mathrm{~mL} / \mathrm{min}$ and the rotation of the cutting disc was 400 - $800 \mathrm{rpm}$. Samples stored for 2 $\mathrm{h}$ after homogenization showed better microencapsulation yield ( 83 - $84 \%$ ) and bead size $(1.07 \mathrm{~mm})$ than those stored for $24 \mathrm{~h}$ after homogenization $(79 \%$ and $1.18 \mathrm{~mm})$. When the loading capacity increased from $20 \%$ to $40 \%$, the mole ratio $\left(\mathrm{CaCl}_{2}\right.$-alginate) in the bead was reduced from 1.55 to 0.86 . This mole ratio data can be considered to control and adjust $\mathrm{CaCl}_{2}$ concentration during the continuous operation of the Jetcutter.
\end{abstract}

Cited as: Truong, V., Ta, P. N. M., \& Nguyen, P. T. (2020). Microencapsulation of Gac (Momordica cochinchinensis Spreng.) fruit oil in Ca-Alginate bead produced through Jetcutter. The Journal of Agriculture and Development 19(4), 80-89. 


\title{
Vi bọc dầu gấc (Momordica cochinchinensis Spreng.) trong hạt Ca-Alginate sản xuất từ thiết bị tia cắt
}

\author{
Trương Vĩnh*, Tạ Ngọc Minh Phương \& Nguyễn Thanh Phương \\ Bộ môn Công Nghệ Hóa Học, Trường Đại Học Nông Lâm TP.HCM, TP. Hồ Chí Minh
}

\section{THÔNG TIN BÀI BÁO}

Bài báo khoa học

Ngày nhận: 07/06/2020

Ngày chỉnh sửa: 22/07/2020

Ngày chấp nhận: 14/08/2020

\section{Từ khóa}

Alginate

Dầu Gấc

Momordica cochinchinensis Spreng.

Thiết bị tia cắt

Vi bọc

*Tác giả liên hệ

Trương Vĩnh

Email: tv@hcmuaf.edu.vn

\section{TÓM TẮT}

Dầu gấc (Momordica cochinchinensis Spreng.) được vi bọc trong hạt Ca-Alginate bằng thiết bị tia cắt. Các thí nghiệm cho thấy cách chuẩn bị nhũ tương và các thông số thiết bị ảnh hưởng có ý nghĩa đến kích thước hạt, hiệu suất vi bọc, năng suất tải và hiệu suất thu hồi của thiết bị. Ở nồng độ $2.5 \%$ alginate, hạt có dạng cầu với kích thước $1,07-1,18 \mathrm{~mm}$ khi lưu lượng dịch $14 \mathrm{~mL} / \mathrm{p}$ và tốc độ đĩa cắt $400-800 \mathrm{v} / \mathrm{p}$. Mẫu trữ 2 giờ sau đồng hóa cho hiệu suất vi bọc $(83-84 \%)$ và kích thước hạt $(1,07 \mathrm{~mm})$ tốt hơn mẫu trữ 24 giờ sau đồng hóa $(79 \%$ và $1,18 \mathrm{~mm})$. Khi tăng năng suất tải $20-40 \%$, tỉ lệ mole $\left(\mathrm{CaCl}_{2}\right.$-alginate) trong hạt vi bọc giảm từ 1,55 xuống 0,86 . Số liệu tỉ lệ mole này có thể tham khảo để kiểm soát và điều chỉnh nồng độ $\mathrm{CaCl}_{2}$ trong quá trình hoạt động liên tục của thiết bị tia cắt.

\section{1. Đặt Vấn Đề}

Công nghệ vi bọc hoạt chất được ứng dụng nhiều trong các lĩnh vực dược phẩm để kiểm soát tốc độ phóng thích thuốc, làm giàu dưỡng chất cho thực phẩm, để tránh oxy hóa hoạt chất trong mỹ phẩm. Vi bọc theo phương pháp ion-gel (ionotropic gelation) được thực hiện bằng cách hòa tan hay khuếch tán hoạt chất trong polymer điện tích âm và nhỏ giọt vào bể chứa ion dương của kim loại kiềm để tạo hạt có liên kết ion. Vì hạt Alginate-Ca ${ }^{2+}$ có bề mặt nhẵn, độ đồng đều cao (Fundueanu \& ctv., 1999) nên alginate là polymer được ứng dụng rộng rãi.

Nguyên lý tạo hạt kiểu nhỏ giọt (dropping methods) có thể được thực hiện bằng tạo từng giọt dùng vòi tiêm, tĩnh điện (electrostatic), rung dao động (vibrational jet-breakup) và thiết bị tia cắt (jet-cutter). Phương pháp nhỏ giọt dùng vòi tiêm cho kích thước hạt 1,5 mm (Truong \& ctv., 2020) hoặc $2-3$ mm (Chan \& ctv., 2009) tùy theo cấu trúc của alginate, ít dùng trong công nghiệp do năng suất thấp và chỉ áp dụng cho dịch có độ nhớt dưới $200 \mathrm{mPas}$ (Prüße \& ctv., 2002). Phương pháp tĩnh điện cho hạt kích thước $0,3-5 \mathrm{~mm}$ và cũng có khuyết điểm như kiểu vòi tiêm (Prüße \& ctv., 2002; Martins \& ctv., 2010). Rung dao động là phương pháp tinh vi nhất của nguyên lý nhỏ giọt (Prüße \& ctv., 2002) nhưng khó sử dụng cho dịch nhớt trên $200 \mathrm{mPas}$ vì hạt có hình thái xấu (Prüße \& ctv., 2008) và thiết bị phức tạp, giá thành cao.

Kích thước hạt lớn và năng suất thấp là những hạn chế của phương pháp nhỏ giọt (Xiao \& ctv., 2013). Để giải quyết hạn chế này, thiết bị tia cắt đã được Prüße \& ctv. (2000) phát triển, cho kích thước hạt từ $0,15-3 \mathrm{~mm}$, tỉ lệ tốc độ bơm/tốc độ cắt và góc nghiêng vòi phun ảnh hưởng đến chất lượng và thu hồi hạt. So với các loại tạo giọt khác, năng suất kiểu tia cắt gấp 12 lần kiểu đĩa quay và 8 lần kiểu rung. Paulo \& ctv. (2017) so sánh thiết bị nhỏ giọt và tia cắt cho thấy muốn 
hạt nhỏ thì phải dùng loại tia cắt, khi tăng từ 200 dến $900 \mathrm{v} / \mathrm{p}$ kích thước hạt giảm từ $5,2 \mathrm{~mm}$ đến $1,1 \mathrm{~mm}$.

Tuy nhiên, các nghiên cứu của Prüße \& ctv. (2000, 2008) và Paulo \& ctv. (2017) chủ yếu khảo sát ảnh hưởng của thông số thiết bị đến kích thước, hình dáng hạt và hiệu suất thu hồi. Chưa thấy báo cáo về ảnh hưởng của quá trình chuẩn bị nhũ tương cùng với các thông số của thiết bị lên chất lượng vi bọc và hiệu suất thu hồi. Do vậy, mục tiêu của nghiên cứu này là khảo sát ảnh hưởng của phương pháp xử lý trước khi tạo hạt như thời gian làm lạnh mẫu sau đồng hóa, tỉ lệ của hoạt chất trong dịch, phương pháp đồng hóa và tốc độ quay của đĩa cắt lên chất lượng vi bọc và hiệu suất thu hồi của thiết bị tia cắt, với dầu gấc là hoạt chất vi bọc.

\section{Vật Liệu và Phương Pháp Nghiên Cứu}

\subsection{Vật liệu}

Dầu gấc của công ty Gacviet Fruit, Việt nam. Sodium Alginate, Sodium citrate, $\mathrm{CaCl}_{2}$ và hexan của Trung Quốc, trong đó Sodium Alginate (Yantai Zhouji) có 2,4\% protein và ít tạp chất đã được kiểm tra trong nghiên cứu trước (Truong \& ctv., 2020).

\subsection{Thiết bị}

Thiết bị tia cắt bao gồm bơm dịch Alginate từ thùng chứa qua vòi (một hoặc nhiều vòi) tạo dòng tia chảy xuống (Hình 1). Một đĩa cắt gồm roto và các dây cắt quay quanh trục để cắt dòng tia thành những giọt nhỏ rơi xuống bể chứa $\mathrm{CaCl}_{2}$. Phản ứng giữa Alginate và $\mathrm{CaCl}_{2}$ sẽ tạo hạt vi bọc.

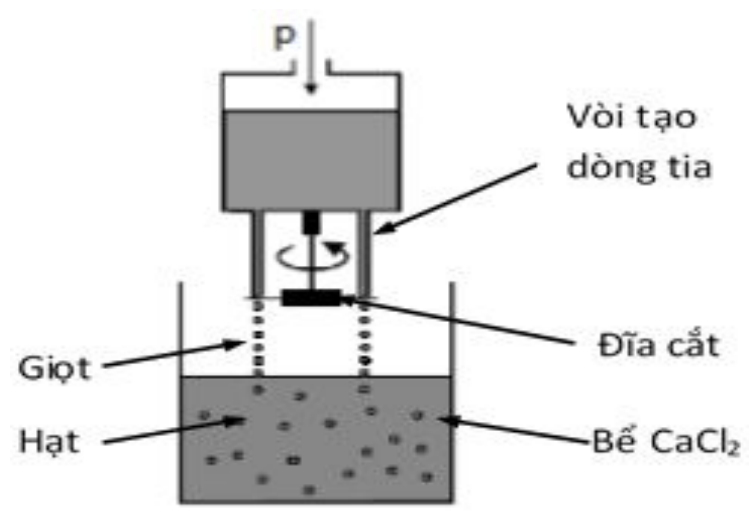

Hình 1. Thiết bị tia cắt.

\subsection{Phương pháp thí nghiệm}

\subsubsection{Tạo nhũ tương}

Áp dụng phương pháp Soliman \& ctv. (2013) có cải biên. Pha $100 \mathrm{~mL}$ Alginate nồng độ $2.5 \%$ trong nước cất và trữ 24 giờ ở $17^{\circ} \mathrm{C}$. Cho dầu Gấc từ từ vào bình alginate (có hòa tan Tween 80 theo $1 \%$ dầu Gấc), khuấy từ $300 \mathrm{v} / \mathrm{p}$. Lượng dầu cho vào $(\mathrm{m} 0)$ được thay đổi theo nồng độ tỉ lệ với lượng alginate $\mathrm{mA}$ để có năng suất tải pha chế $\mathrm{NST}_{0}$ cho trước theo (1):

$$
\mathrm{NST}_{0} \%=\mathrm{m}_{0} /\left(\mathrm{m}_{0}+\mathrm{mA}\right) * 100
$$

Hệ nhũ tương được đồng hóa $13500 \mathrm{v} / \mathrm{p}$ bằng máy khuấy cơ học 3 phút hoặc siêu âm 5 phút ở công suất $45 \mathrm{~W}$. Mẫu sau đồng hóa được trữ lạnh qua đêm $\left(17^{\circ} \mathrm{C}\right)$ gọi là mẫu lạnh; không để qua đêm gọi là mẫu không lạnh, trước khi vi bọc sẽ đưa nhiệt độ mẫu về $26^{\circ} \mathrm{C}$.

\subsubsection{Tạo hạt vi bọc}

Bơm nhũ tương Alginate-dầu Gấc qua kim tiêm bằng thiết bị tia cắt với lưu lượng $14 \mathrm{~mL} / \mathrm{p}$ tạo ra dòng tia và được đĩa cắt thành các giọt rơi vào bình chứa $\mathrm{CaCl}_{2} \quad 0,5 \%$ (15 mM). Khuấy từ dịch $\mathrm{CaCl}_{2}$ liên tục, thời gian phản ứng 25 phút. Cho hạt ra vợt để trên giấy thấm 10 phút để xác định kích thước, hiệu suất vi bọc, năng suất tải thực tế (NST), hiệu suất thu hồi chất rắn, năng suất tải riêng LCR. Mẫu đối chứng thực hiện tương tự nhưng không có đĩa cắt.

\subsection{3. Đo kích thước hạt vi bọc}

Đo kích thước hạt bằng phương pháp hình ảnh và xử lý bằng phần mềm ImageJ-1.52r. Đường kính tương đương từng hạt di được xác định bằng phần mềm và đường kính trung bình Sauter $\mathrm{d} 3,2$ từ 30 hạt được tính theo công thức (2) (Mohsenin, 1970)

$$
\mathrm{d}_{3,2}=\frac{d_{1}^{3}+d_{2}^{3}+\ldots+d_{\mathrm{n}}^{3}}{d_{1}^{2}+d_{2}^{2}+\ldots+d_{\mathrm{n}}^{2}}
$$

\subsubsection{Phân tích dầu Gấc bằng quang phổ}

Pha dầu Gấc trong hexan theo các nồng độ khác nhau. Để tìm bước sóng cho độ hấp thu cực đại, mẫu pha $0,02 \mathrm{~mL}$ dầu/10 mL hexan được quét ở bước 340 - $600 \mathrm{~nm}$. Thiết bị sử dụng là máy quang phổ Hewlett Packard UV-Vis. Dựng 
đường chuẩn hấp thu dầu Gấc ở bước sóng hấp thu cực đại.

\subsubsection{Xác định hiệu suất vi bọc MEY}

Cân 1 gram hạt tươi (khoảng $0,5 \mathrm{~g}$ ), cho vào $5 \mathrm{~mL}$ dịch citrate $0,055 \mathrm{M}$, votex đến tan, cho $5 \mathrm{~mL}$ hexan vào và votex trong 3 phút. Ly tâm $6000 \mathrm{v} / \mathrm{p}$ trong 10 phút tách lớp, lấy hexan ở trên đo quang phổ, tính được lượng dầu vi bọc $\mathrm{m}_{0}$ dựa vào đường chuẩn. Hiệu suất vi bọc tính theo công thức sau, với mi là lượng dầu ban đầu trong nhũ tương Alginate- $\mathrm{CaCl}_{2}$.

$$
\operatorname{MEY}(\%)=\mathrm{m}_{0} / \mathrm{m}_{\mathrm{i}} \mathrm{x} 100
$$

2.3.6. Hiệu suất thu hồi chất rắn YR

$$
\mathrm{Y}_{\mathrm{R}}(\%)=\frac{\text { khối lượng hạt thu được } \mathrm{x} 100}{\text { khối lượng nguyên liệu ban đầu }}
$$

\subsubsection{Năng suất tải thực tế NST}

Năng suất tải thực tế NST là tỉ số giữa lượng dầu vi bọc và khối lượng chất khô của hạt, tính theo công thức (5), W là ẩm độ hạt (căn bản ướt).

$$
\mathrm{NST}=\mathrm{m}_{0} /(1-\mathrm{W}) \mathrm{m}_{1}
$$

\subsubsection{Bố trí thí nghiệm}

Các thí nghiệm bố trí theo Bảng 1. Trong đó, LCR là tỉ số giữa NST và năng suất tải pha chế $\mathrm{NST}_{0}$. Ký hiệu L là "mẫu lạnh', KL là mẫu "không lạnh", C là "đồng hóa cơ học" và SA là đồng hóa siêu âm. Ví dụ, mẫu 30LC400 có $\mathrm{NST}_{0}=30 \%$, mẫu lạnh $(\mathrm{L})$, đồng hóa cơ $(\mathrm{C})$, số vòng quay đĩa $400 \mathrm{v} / \mathrm{p}$. Thí nghiệm được lặp lại 3 lần, số liệu được xử lý thống kê bằng phần mềm Statgraphic Centurion v15.1 và Excel.

\section{Kết Quả và Thảo Luận}

\subsection{Chế tạo thiết bị}

Thiết bị tia cắt được thiết kế, chế tạo sử dụng trong nghiên cứu là một phần đề tài cấp Bộ B2018-NLS13 cho trên Hình 2 và 3 (Truong, 2020). Số vòng quay đĩa cắt có thể điều chỉnh vô cấp tối đa $10000 \mathrm{v} / \mathrm{p}$. Bơm dịch có áp suất tối đa $3 \mathrm{kgf} / \mathrm{cm}^{2}$, lưu lượng điều chỉnh vô cấp. Trong nghiên cứu này dùng kim phun đường kính 0,26 $\mathrm{mm}$.

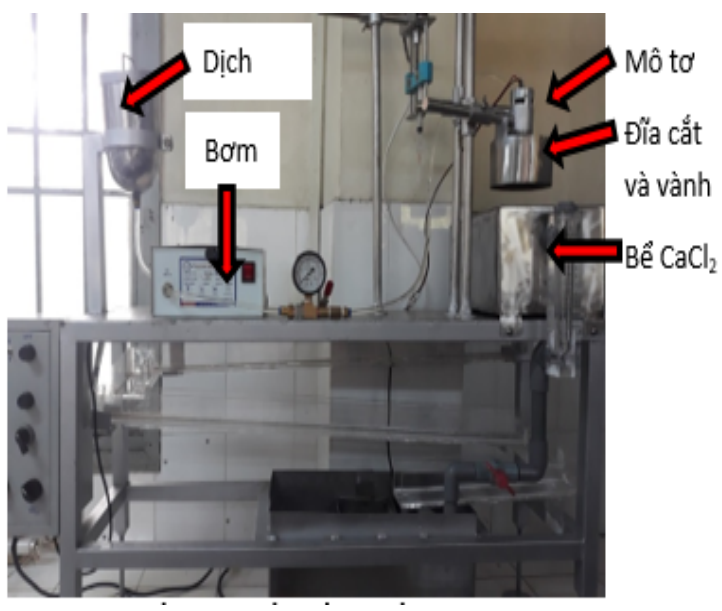

Hình 2. Thiết bị tia cắt (đề tài cấp Bộ B2018 NLS13).

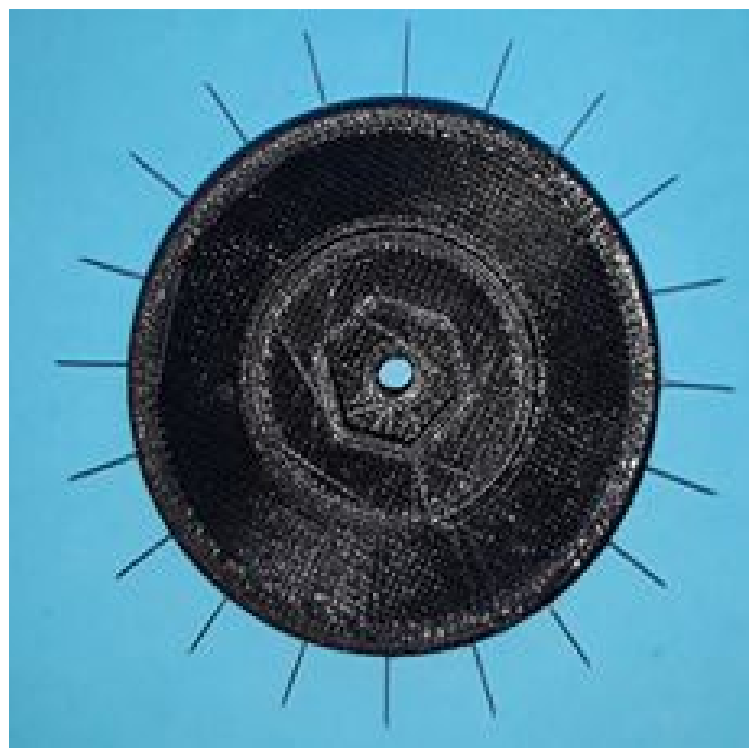

Hình 3. Cấu tạo đĩa cắt, gồm roto và 24 dây cắt $\mathrm{d}_{\mathrm{w}}$ $=0,31 \mathrm{~mm}$.

\subsection{Kết quả thí nghiệm}

Chọn bước sóng và dựng đường chuẩn: độ hấp thu dầu gấc trong hexan ở các bước sóng khác nhau cho trên Hình 4. Độ hấp thu cực đại tại $447 \mathrm{~nm}$, thể hiện sự hấp thu của beta-carotene ở 448 nm của nhiều tác giả (Tan \& Soderstrom, 1989; Mercadante \& Amaya, 1998), và ở 450 nm (Abdul-Hammed, 2013). Lycopene thể hiện peak không rõ ở bước 470 - 472 nm do hàm lượng trong mẫu ít. Trong nghiên cứu này, bước $447 \mathrm{~nm}$ được chọn để phân tích dầu gấc trong các mẫu hạt vi bọc. 
Bảng 1. Thiết kế thí nghiệm

\begin{tabular}{ccccc}
\hline Mẫu & NST $_{0} \%$ & Xử lý & Đồng hóa & Số vòng quay đĩa $(\mathrm{v} / \mathrm{p})$ \\
\hline 20LC400 & 20,0 & Lạnh & Cơ & 400 \\
30LC400 & 30,0 & Lạnh & Cơ & 400 \\
40LC400 & 40,0 & Lạnh & Cơ & 400 \\
30LC600 & 30,0 & Lạnh & Cơ & 600 \\
30LC800 & 30,0 & Lạnh & Cơ & 800 \\
30KLC400 & 30,0 & Không lạnh & Cơ & 400 \\
30KLC600 & 30,0 & Không lạnh & Cơ & 400 \\
30KLSA400 & 30,0 & Không lạnh & Siêu âm & 400 \\
\hline
\end{tabular}

Bảng 2. Kết quả thí nghiệm

\begin{tabular}{cccccc}
\hline Mẫu & NST $_{0} \%$ & MEY, $\%$ & $\mathrm{Y}_{\mathrm{R}}, \%$ & $\mathrm{NST}, \%$ & $\mathrm{LC}_{\mathrm{R}}$ \\
\hline 20LC400 & 20,0 & $77,3 \pm 1,96^{\mathrm{c}}$ & $54,3 \pm 0,78^{\mathrm{c}}$ & $10,9^{\mathrm{a}} \pm 0,25$ & $0,55^{\mathrm{a}} \pm 0,04$ \\
30LC400 & 30,0 & $79,0 \pm 0,42^{\mathrm{c}}$ & $56,6 \pm 0,93^{\mathrm{c}}$ & $19,1^{\mathrm{b}} \pm 0,10$ & $0,64^{\mathrm{b}} \pm 0,03$ \\
40LC400 & 40,0 & $79,2 \pm 0,66^{\mathrm{c}}$ & $54,6 \pm 1,71^{\mathrm{c}}$ & $29,9^{\mathrm{c}} \pm 0,25$ & $0,75^{\mathrm{c}} \pm 0,04$ \\
30LC600 & 30,0 & $76,8 \pm 1,63^{\mathrm{b}}$ & $38,2 \pm 1,09^{\mathrm{b}}$ & $19,6^{\mathrm{b}} \pm 0,45$ & $0,65^{\mathrm{b}} \pm 0,06$ \\
30LC800 & 30,0 & $72,5 \pm 0,50^{\mathrm{a}}$ & $8,4 \pm 0,93^{\mathrm{a}}$ & $18,9^{\mathrm{b}} \pm 0,65$ & $0,63^{\mathrm{b}} \pm 0,07$ \\
30KLC400 & 30,0 & $83,6 \pm 0,04^{\mathrm{e}}$ & $54,6 \pm 0,60^{\mathrm{c}}$ & $21,3^{\mathrm{b}} \pm 0,23$ & $0,71^{\mathrm{b}} \pm 0,04$ \\
30KLC600 & 30,0 & $84,5 \pm 1,15^{\mathrm{e}}$ & $55,9 \pm 1,10^{\mathrm{c}}$ & $20,4^{\mathrm{b}} \pm 0,31$ & $0,68^{\mathrm{b}} \pm 0,05$ \\
30KLSA400 & 30,0 & $82,9 \pm 1,33^{\mathrm{d}}$ & $58,0 \pm 0,80^{\mathrm{d}}$ & $21,1^{\mathrm{b}} \pm 0,26$ & $0,70^{\mathrm{b}} \pm 0,04$ \\
\hline
\end{tabular}

${ }^{\mathrm{a}-\mathrm{e}}$ Các số trung bình trên một cột có chỉ số khác nhau thì khác biệt có ý nghĩa thống kê $(P<0.05)$.

Số liệu thực nghiệm: Các kết quả theo thiết kế cho trên Bảng 2.

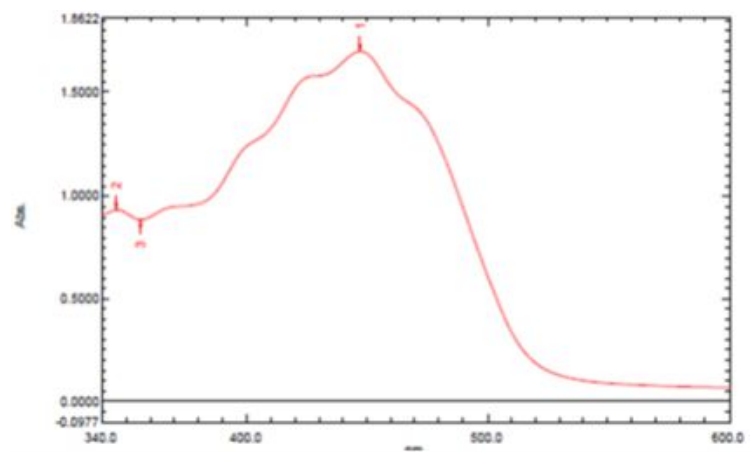

Hình 4. Phổ hấp thu dầu gấc ở các bước sóng khác nhau. Cực đại ở peak 1 (447 nm) thể hiện sự hấp thu của beta-carotene. Peak 2 là isomer của betacarotene.

\subsection{Thảo luận}

\subsection{1. Ảnh hưởng phương pháp tạo hạt lên hình thái và kích thước hat}

Hình thái hạt các mẫu alginate 2,5\% làm từ thiết bị đĩa cắt, có dạng cầu khá đều và giống nhau. Không có khác biệt về hình thái giữa lạnh và không lạnh, và giữa các số vòng quay từ 400
- 800 v/p (Hình 5). Phân bố kích thước hạt có dạng hình chuông ở các số vòng quay $\mathrm{n}=400$ v/p - 800 v/p (Hình 6).

Ở lưu lượng $14 \mathrm{~mL} / \mathrm{p}$, khi tăng $\mathrm{n}$ từ $400 \mathrm{v} / \mathrm{p}$ lên $800 \mathrm{v} / \mathrm{p}$ thì kích thước trung bình hạt không đổi $(1,178-1,156 \mathrm{~mm}, P>0,05$, Bảng 3$)$. Theo Paulo \& ctv. (2017), số vòng quay có ảnh hưởng đến kích thước hạt, cụ thể ở $\mathrm{n}=300 \mathrm{v} / \mathrm{p}, 600$ $\mathrm{v} / \mathrm{p}$ và $900 \mathrm{v} / \mathrm{p}$ thì kích thước hạt lần lượt 3,8 $\mathrm{mm}, 1,5 \mathrm{~mm}$ và $1,1 \mathrm{~mm}$. Ở trong nghiên cứu này, tăng số vòng quay chỉ thấy rõ sự giảm độ đồng đều hạt thể hiện qua sự tăng số span. Mẫu lạnh ở $400 \mathrm{v} / \mathrm{p}$ có độ đồng đều hạt cao nhất với span là 0,343 so với $800 \mathrm{v} / \mathrm{p}(\operatorname{span}=0,556)$.

Ở 30\% dầu Gấc, mẫu không lạnh có kích thước $1,07 \mathrm{~mm}$ thấp hơn so với mẫu lạnh là $1,18 \mathrm{~mm}$ $(P<0,05)$. Có thể giải thích là thời gian xử lý mẫu lạnh dài nên bài khí triệt để dẫn đến độ nhớt cao khó cắt đứt làm cho kích thước hạt lớn hơn mẫu không lạnh. Thiết bị tia cắt chế tạo trong đề tài này cho hạt kích thước $(1,07 \mathrm{~mm})$ đã giảm hơn so với nhỏ giọt là $1,5 \mathrm{~mm}$ (Truong \& ctv., 2020) hoặc 2 mm (Chan \& ctv., 2009). Khi tăng số dây cắt và số vòng quay lên, tần số cắt cao làm giảm kích thước hạt. Ví dụ từ thiết bị có $\mathrm{Z}=48$ dây cắt, $\mathrm{n}=4500 \mathrm{v} / \mathrm{p}$ (Prüße \& ctv., 2002, 2008) kích thước hạt trung bình $0,77 \mathrm{~mm}$ và phân bố 


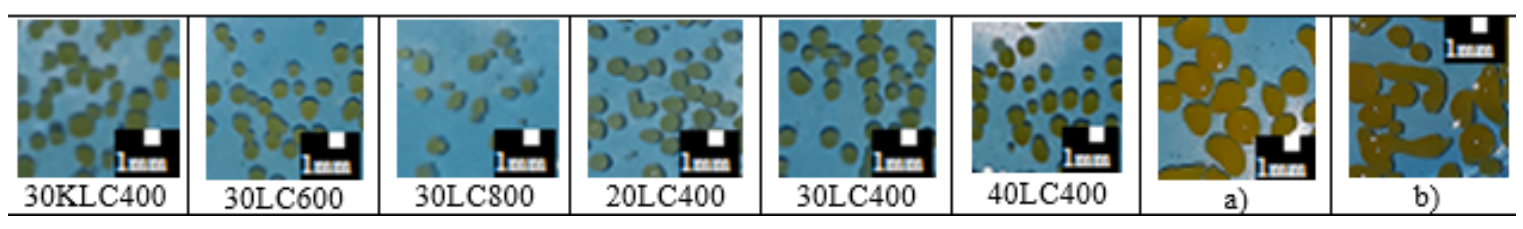

Hình 5. Hình thái hạt của các mẫu đồng hóa cơ học, làm từ thiết bị đĩa cắt lưu lượng $14 \mathrm{~mL} / \mathrm{p}$. Mẫu đối chứng $\mathrm{a}$ ) và $\mathrm{b}$ ) làm từ kiểu dòng tia không có đĩa cắt, $\mathrm{NST}_{0}=30 \%$, lưu lượng $4 \mathrm{~mL} / \mathrm{p}$ và $14 \mathrm{~mL} / \mathrm{p}$, theo thứ tự.

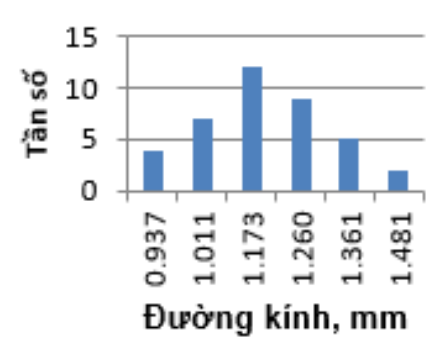

a) $\mathrm{n}=400 \mathrm{v} / \mathrm{p}$
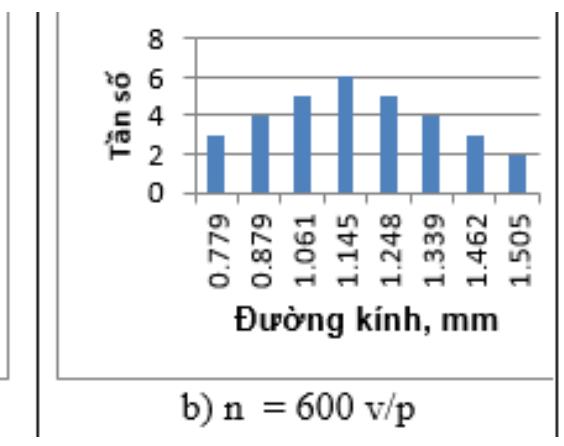

b) $\mathrm{n}=600 \mathrm{v} / \mathrm{p}$

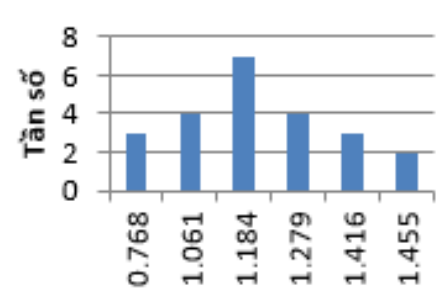

Đường kính, mm

c) $\mathrm{n}=800 \mathrm{v} / \mathrm{p}$

Hình 6. Phân bố kích thước hạt mẫu lạnh, đồng hóa cơ học, năng suất tải $30 \%$.

Bảng 3. Thiết kế thí nghiệm

\begin{tabular}{ccccc}
\hline Mẫu & $\mathrm{d}_{\mathrm{p}}(\mathrm{mm})$ & $\mathrm{Span}$ & $\mathrm{d}_{\mathrm{pmin}}(\mathrm{mm})$ & $\mathrm{d}_{\mathrm{pmax}}(\mathrm{mm})$ \\
\hline 20LC400 & $1,180^{\mathrm{a}} \pm 0,014$ & 0,30 & $0,918^{\mathrm{c}} \pm 0,011$ & $1,33^{\mathrm{a}} \pm 0,014$ \\
30LC400 & $1,178^{\mathrm{a}} \pm 0,019$ & 0,34 & $0,848^{\mathrm{b}} \pm 0,017$ & $1,54^{\mathrm{b}} \pm 0,018$ \\
40LC400 & $1,157^{\mathrm{a}} \pm 0,020$ & 0,44 & $0,605^{\mathrm{a}} \pm 0,020$ & $1,43^{\mathrm{ab}} \pm 0,019$ \\
30LC600 & $1,160^{\mathrm{a}} \pm 0,023$ & 0,57 & $0,71^{\mathrm{ab}} \pm 0,021$ & $1,51^{\mathrm{b}} \pm 0,021$ \\
30LC800 & $1,156^{\mathrm{a}} \pm 0,024$ & 0,56 & $0,69^{\mathrm{ab}} \pm 0,020$ & $1,46^{\mathrm{ab}} \pm 0,023$ \\
30KLC400 & $1,082^{\mathrm{b}} \pm 0,018$ & 0,41 & $0,77^{\mathrm{ab}} \pm 0,022$ & $1,30^{\mathrm{a}} \pm 0,017$ \\
30KLC600 & $1,078^{\mathrm{b}} \pm 0,019$ & 0,51 & $0,76^{\mathrm{ab}} \pm 0,021$ & $1,33^{\mathrm{a}} \pm 0,018$ \\
30KLSA400 & $1,091^{\mathrm{b}} \pm 0,017$ & 0.21 & $0,75^{\mathrm{ab}} \pm 0,018$ & $1,35^{\mathrm{a}} \pm 0,016$ \\
\hline a-c Giá tri trung bình có ký tư khác nhau thì khác biệt có ý nghĩa thống kê $(P<0,05)$.
\end{tabular}

hạt từ $0,3 \mathrm{~mm}$ đến $2 \mathrm{~mm}$. Ở trong đề tài này, phân bố hạt làm từ thiết bị đĩa cắt là $0,71-1,54$ mm (Bảng 3).

Năng suất tải ảnh hưởng đáng kể đến kích thước nhỏ nhất $\left(\mathrm{d}_{\mathrm{pmin}}\right)$ và lớn nhất $\left(\mathrm{d}_{\mathrm{pmax}}\right)$. Ở $400 \mathrm{v} / \mathrm{p}$, khi tăng năng suất tải từ $20 \%$ lên $40 \%$ thì kích thước trung bình $1,16-1,18 \mathrm{~mm}$ nhưng kích thước dpmin giảm từ $0,92 \mathrm{~mm}$ xuống 0,61 mm $(P<0,05)$. Với mẫu không lạnh, kích thước hạt chế độ đồng hóa cơ học $(1,076 \mathrm{~mm})$ và siêu âm $(1,096 \mathrm{~mm})$ tương đương nhau nhưng mẫu siêu âm có phân bố hạt ( $\operatorname{span}=0,207)$ đồng đều hơn đồng hóa cơ ( $\operatorname{span}=0,414$, Bảng 3$)$. Sự giảm kích thước trung bình của mẫu không lạnh so với mẫu lạnh $(\mathrm{n}=400 \mathrm{v} / \mathrm{p}$ và $600 \mathrm{v} / \mathrm{p})$ chủ yếu là do giảm kích thước dpmax. Kích thước hạt càng nhỏ thì hạt vi bọc càng dễ ứng dụng do phân phối hoạt chất đồng đều hơn (Chan, 2011; Prüße \& ctv., 2000).

Phương pháp dòng tia không dĩa cắt ở lưu lượng $4 \mathrm{~mL} / \mathrm{p}$ cho hạt $3-4 \mathrm{~mm}$ (Hình $4 \mathrm{a}$ ), khi tăng lưu lượng lên $14 \mathrm{~mL} / \mathrm{p}$ (Hình 4b) tạo ra những sợi dài, hình dạng không ổn định. Theo Prüße \& ctv. (2008), phương pháp rung dòng tia (ví dụ thiết bị B-390 của hãng BUCHI) không thể tạo hạt trên $2 \%$ alginate. Điều này nói lên ưu điểm của phương pháp đĩa cắt so với các phương pháp khác là kích thước hạt nhỏ, năng suất cao với hình thái hạt tròn dù ở nồng độ alginate trên $2 \%$. 


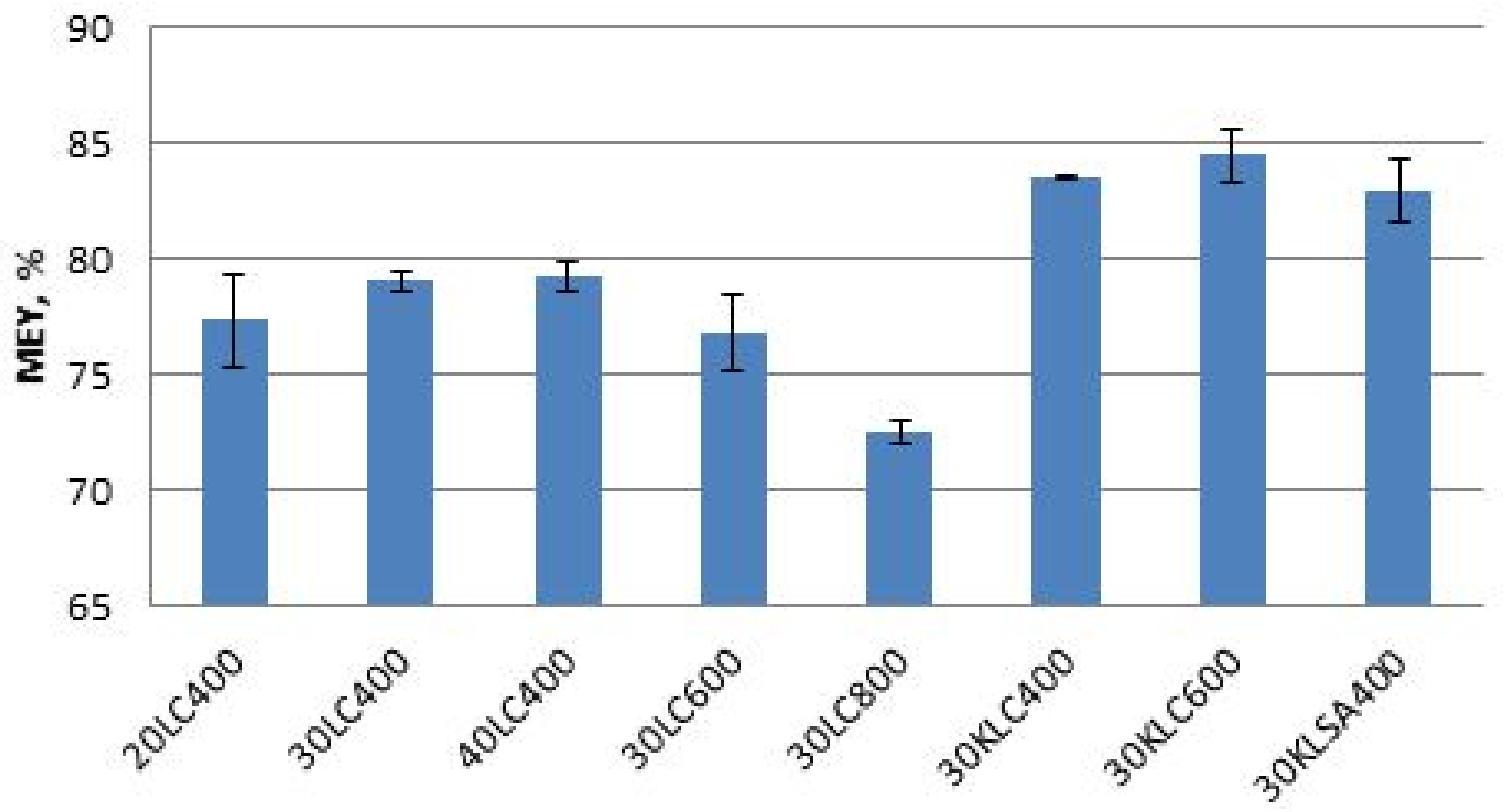

Phương pháp tạo hạt

Hình 7. Hiệu suất vi bọc dầu gấc của các phương pháp tạo hạt khác nhau (kết quả thống kê ở Bảng 2).

\subsection{2. Ảnh hưởng phương pháp tạo hạt lên MEY}

Đối với mẫu lạnh hiệu suất vi bọc MEY (77,3$79,25 \%)$ không bị ảnh hưởng khi tăng nồng độ dầu gấc từ $20 \%$ đến $40 \%(P>0,05)$, nhưng bị giảm khi tăng số vòng quay $(P<0,05)$. MEY cao nhất ở $400 \mathrm{v} / \mathrm{p}(79 \%)$ và thấp nhất ở $800 \mathrm{v} / \mathrm{p}$ $(72,5 \%$, Bảng 2 và Hình 7$)$. Điều này cũng tương tự thu hồi và năng suất tải $(P<0,05)$. Năng suất tải và MEY giảm khi tăng số vòng quay có lẽ do lực cắt lớn làm hao tổn dầu gấc. Ngoài ra, mẫu không lạnh ở năng suất tải $30 \%$ cho hiệu suất vi bọc $(83,6$ - 84,5\%) cao hơn mẫu lạnh. Trong quá trình đồng hóa tạo hệ nhũ tương $\mathrm{o} / \mathrm{w}$ trong đó các giọt dầu gấc được bọc trong chất hoạt động bề mặt Tween 80. Sau khi đồng hóa mẫu trữ lạnh 24 giờ có lẽ một phần dầu gấc đã thoát ra khỏi lớp bọc Tween nên khi nhỏ giọt vào bể $\mathrm{CaCl}_{2}$ thì lượng dầu gấc này dễ thất thoát giải thích tại sao mẫu trữ lạnh qua đêm cho hiệu suất vi bọc thấp hơn mẫu không trữ lạnh. Có sự tương tác giữa phương pháp xử lý mẫu trước khi tạo hạt và số vòng quay khi xem xét đáp ứng MEY và YR. Ở nghiệm thức không lạnh-600 v/p cho MEY cao nhất $(84,5 \%)$ và ở nghiệm thức lạnh-600 v/p cho YR thấp nhất $(38,2 \%)$. Không có sự khác biệt giữa siêu âm và cơ học trên các chỉ tiêu MEY $(P$
$>0,05)$.

\subsection{3. Ảnh hưởng phương pháp tạo hạt lên NST và ${ }^{L} C_{R}$}

Bảng 2 và Hình 8 cho thấy, phương pháp xử lý lạnh và không lạnh có LCR xấp xĩ nhau, $(0,64$ - 0,69). Ở cùng $\mathrm{NST}_{0}$ là $30 \%$, khi tăng số vòng quay từ $400 \mathrm{v} / \mathrm{p}$ lên $800 \mathrm{v} / \mathrm{p}$ không ảnh hưởng đến NST $(P>0,05)$. Khi tăng năng suất tải pha chế NST0 từ $20-40 \%$, tỉ số $\mathrm{LC}_{R}$ tăng $0,55-0,75$ $(P<0,05)$. Năng suất tải pha chế $\left(\mathrm{NST}_{0}\right)$ là tỉ số giữa dầu gấc và (Alginate+ dầu). Còn Năng suất tải thực tế (NST) là tỉ số giữa dầu gấc và $\left(\right.$ alginate + dầu + Tween $\left.+\mathrm{CaCl}_{2}\right)$. Như vậy khi $\mathrm{LC}_{R}$ cao tức hàm lượng Tween và $\mathrm{CaCl}_{2}$ trong hạt vi bọc giảm tương đối. Nghĩa là số mole $\mathrm{Ca}^{2+}$ tham gia liên kết với Alginate giảm. Tuy nhiên, chỉ dựa vào $L C R$ vẫn chưa đủ để giải thích mối liên kết $\mathrm{Ca}^{2+}$ và Alginate. Ta có thể tính toán tỉ lệ mol liên kết giữa $\mathrm{Ca}^{2+}$ và Alginate như sau:

Vì NST $=\mathrm{m}_{0} /\left(\mathrm{m}_{0}+\mathrm{m}_{\mathrm{A}}+\mathrm{m}_{\mathrm{T}}+\mathrm{m}_{\mathrm{Ca}}\right)$, trong đó mẫu số là chất khô hạt ở công thức (4), với $m_{\mathrm{T}}$ và $\mathrm{m}_{\mathrm{Ca}}$ là lượng Tween 80 và $\mathrm{Ca}^{2+}$ tham gia liên kêt, theo thứ tự, tính được $\mathrm{m}_{\mathrm{Ca}}$ theo (6)

$$
\mathrm{m}_{\mathrm{Ca}}=\mathrm{m}_{0}\left(1 / \mathrm{NST}-1 / \mathrm{NST}_{0}\right)-\mathrm{m}_{\mathrm{T}}
$$




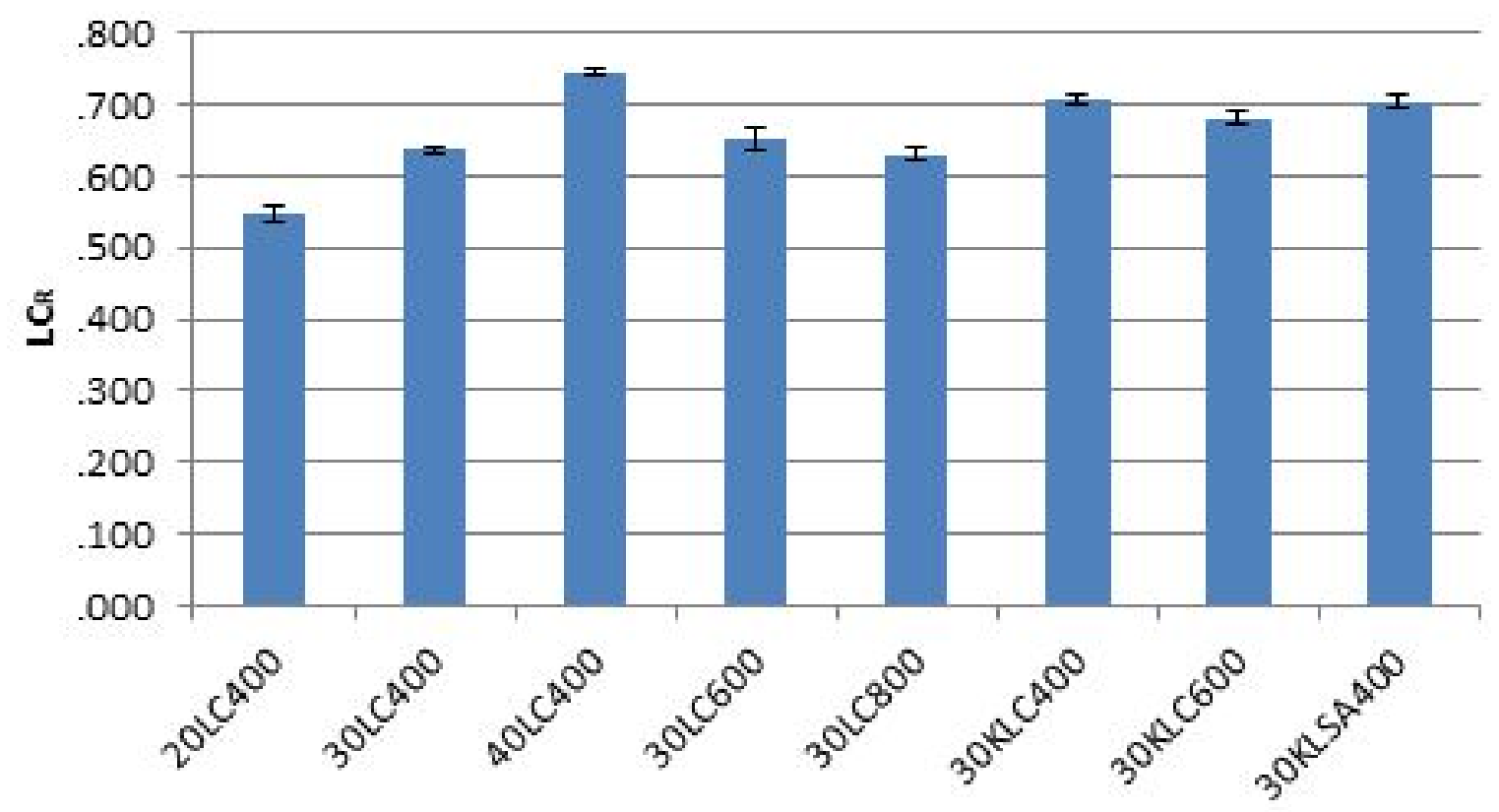

Phương pháp tạo hạt

Hình 8. Hiệu quả năng suất tải của các phương pháp tạo hạt khác nhau (kết quả thống kê ở Bảng 2).

Bảng 4. Tính toán tỉ lệ liên kết mole giữa Calcium và Alginate

\begin{tabular}{cccc}
\hline Mẫu & Mol Ca & Mol Ca/Mol Alg & $\mathrm{Mol} \mathrm{CaCl}_{2} / \mathrm{Mol} \mathrm{Alg}$ \\
\hline 20LC400 & 0,050 & 4,303 & 1,551 \\
30LC400 & 0,040 & 3,458 & 1,246 \\
40LC400 & 0,028 & 2,390 & 0,861 \\
30LC600 & 0,036 & 3,147 & 1,134 \\
30LC800 & 0,038 & 3,245 & 1,169 \\
30KLC400 & 0,030 & 2,626 & 0,946 \\
30KLC600 & 0,035 & 3,031 & 1,092 \\
30KLSA400 & 0,031 & 2,699 & 0,972 \\
\hline
\end{tabular}

Bảng 4 cho thấy số vòng quay và phương pháp đồng hóa không ảnh hưởng đến tỉ lệ mol liên kết. Tuy nhiên, khi tăng $\mathrm{NST}_{0}$ thì tỉ lệ mol $\mathrm{Ca} / \mathrm{mol}$ Alginate giảm dần, phù hợp với sự tăng $\mathrm{LC}_{\mathrm{R}}$ cho trên Hình 8.

Trong kết luận dựa vào $\mathrm{LC}_{\mathrm{R}}$ ở trên (Hình 8) cho thấy mẫu lạnh và không lạnh có $\mathrm{LC}_{\mathrm{R}}$ không thay đổi, nhưng dựa vào số liệu tỉ lệ mol (Bảng 4), ở $400 \mathrm{v} / \mathrm{p}$ và tải $30 \%$, tỉ lệ $\mathrm{mol}(\mathrm{Ca}) / \mathrm{mol}($ Alginate) giảm từ 3,458 (mẫu lạnh) xuống 2,626 (mẫu không lạnh). Bảng 4 còn giúp tính toán số mol $\mathrm{CaCl}_{2}$ tham gia liên kết, đặc biệt quan trọng với hệ thống tạo hạt liên tục, giúp kiểm soát và điều chỉnh nồng độ $\mathrm{CaCl}_{2}$ trong bể $\mathrm{CaCl}_{2}$ khi dòng giọt alginate liên tục chảy xuống. Đây là một thông tin ít thấy công bố trên thế giới và trong nước.

3.3.4. Ảnh hưởng phương pháp tạo hạt lên $Y_{R}$

Ở RPM400 và $\mathrm{NST}_{0} 30 \%$, thu hồi không thay đổi giữa lạnh $(56,6 \%)$ và không lạnh $(54,6 \%$, Bảng 2 và Hình 9$)$. Phân tích ANOVA cho thấy có sự tương tác giữa phương pháp xử lý mẫu trước khi tạo hạt và $\mathrm{RPM}$ khi xem xét đáp ứng $\mathrm{Y}_{\mathrm{R}}$. Phương pháp đồng hóa mẫu không lạnh cho thấy siêu âm làm tăng $\mathrm{Y}_{\mathrm{R}}(58 \%)$ so với cơ học $(54,6 \%)(P<$ $0,05)$. Khi tăng nồng độ dầu gấc từ $20 \%$ dến $40 \%$, thu hồi $\mathrm{Y}_{\mathrm{R}}(54,3-56,6 \%)$ không bị ảnh hưởng $(P$ $>0.05)$. Với phương pháp đồng hóa cơ học, thu 


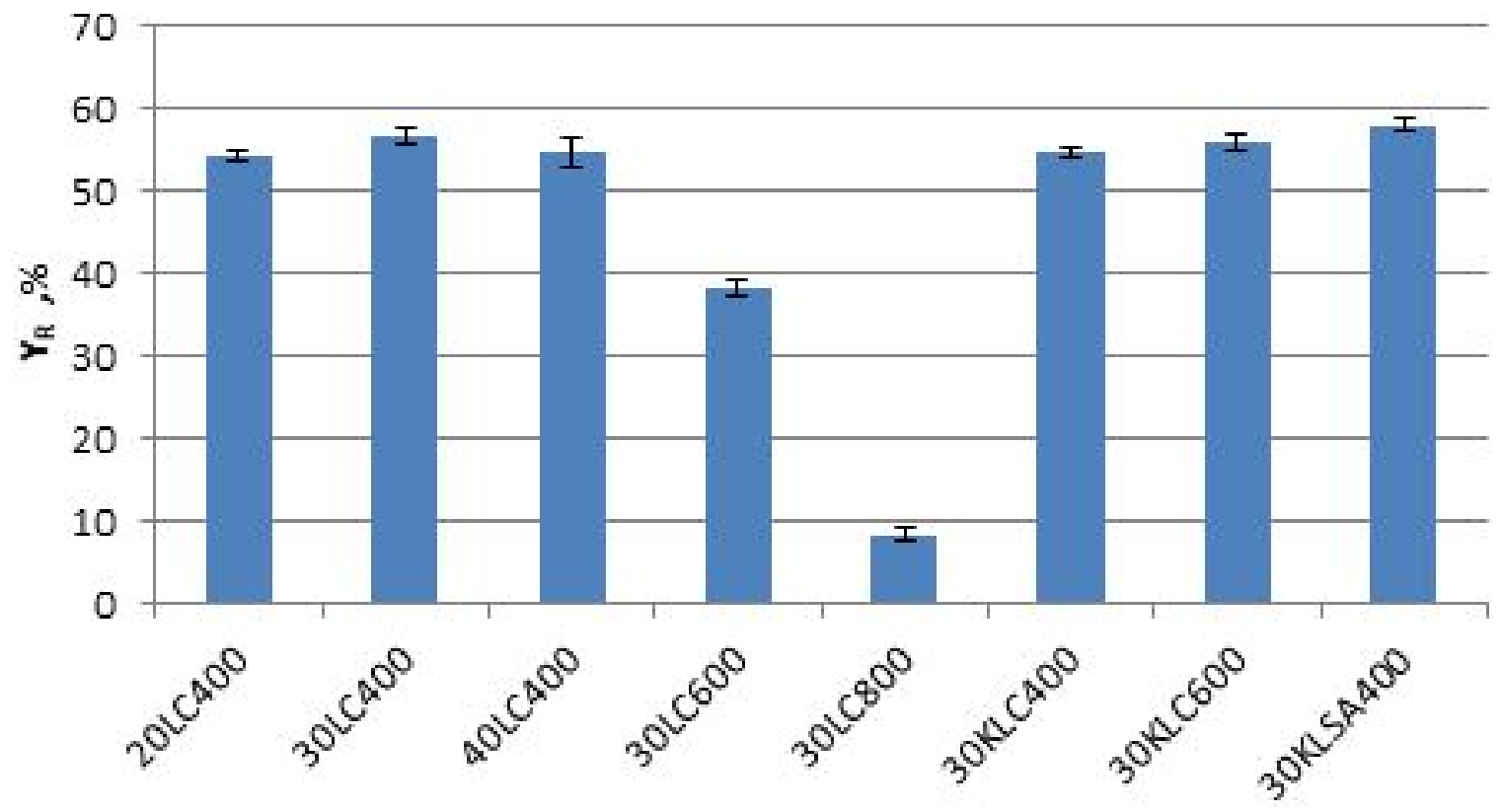

Phương pháp tạo hạt

Hình 9. Tỉ lệ thu hồi chất rắn của các phương pháp tạo hạt khác nhau nhau (kết quả thống kê ở Bảng 2).

hồi cao nhất ở RPM400 (56,6\%) và thấp nhất ở RPM800 (8,4\%). Tổn thất còn cao (trên $53 \%$ ) do hạt bị lực cắt mạnh đẩy văng ra xa vùng hứng hạt (bề $\left.\mathrm{CaCl}_{2}\right)$. Khi tăng số vòng quay, thu hồi càng thấp do lực cắt mạnh hơn. Tổn thất thấp nhất của thiết bị hiện tại là $43,4 \%$. Tổn thất này có thể cải thiện bằng cách điều chỉnh bể hứng hạt.

\section{Kết Luận}

Thiết bị đĩa cắt (Jetcutter) đã hoạt động ổn định tạo được hạt vi bọc dầu gấc ở nồng độ $2,5 \%$ alginate có kích thước $(1,07 \mathrm{~mm})$ nhỏ hơn kiểu dòng tia không đĩa cắt (dripping method, $\mathrm{d}=3$ $4 \mathrm{~mm}$ ), nhỏ hơn kiểu nhỏ giọt (dropping method, $\mathrm{d} \geq 1,5 \mathrm{~mm}$ ), và có năng suất vượt trội. Các mẫu không lạnh ở nồng độ alginate $2,5 \%$ với $\mathrm{n}=400$ - 600 v/p có MEY cao nhất, NST cao nhất, thu hồi hạt $Y_{R}$ cao nhất, kích thước hạt bé nhất, độ đồng đều kích thước cao nhất nên là mẫu tốt nhất của thiết bị khảo sát. Thiết bị tia cắt ở nồng độ alginate cao trên $2 \%$ có ưu điểm so với các dạng tạo hạt khác về năng suất và hình thái hạt. Việc tính toán xấp xỉ tỉ lệ mole của $\mathrm{CaCl}_{2}$ và Alginate theo thông số tạo hạt định hướng cho việc điều chỉnh nồng độ $\mathrm{CaCl}_{2}$ trong thiết kế thiết bị vi bọc tia cắt hoạt động ở chế độ liên tục.

\section{Tài Liệu Tham Khảo (References)}

Abdul-Hammed, M., Bello, I, D., \& Oladoye, S. O. (2013). Simultaneous spectrophotometric determination of lycopene and beta-carotene concentrations in carotenoid mixtures of the extract of tomatoes, papaya and orange juice. Pakistan Journal of Scientific and Industrial Research 56(2), 90-97.

Chan, E. S. (2011). Preparation of Ca-Alginate beads contain-ing high oil content: Influence of process variables on encapsulation efficiency and bead properties. Carbo-hydrate Polymers 84(4), 1267-1275.

Chan, E. S., Lee, B.-B., Ravindra, P., \& Poncelet, D. (2009). Prediction models for shape and size of ca-alginate macrobeads produced through extrusion-dripping method. Journal of Colloid and Interface Science 338, 63-72.

Fundueanu, G., Nastruzzi, C., Carpov, A., Desbrieres, J., \& Rinaudo, M. (1999). Physico-chemical characterization of Ca-alginate microparticles produced with different methods. Biomaterials 20(15), 1427-1435.

Martins, I. M., Maria, F. B., Manuel, C., \& Alírio, E. R. (2014). Microencapsulation of essential oils with biodegradable polymeric carriers for cosmetic applications. Chemical Engineering Journal 245, 191-200. 
Mercadante, A. Z., \& Rodriguez-Amaya, D. B. (1998). Effects of ripening, cultivar differences, and processing on the carotenoid composition of mango. Journal of Agricultural Food Chemistry 46, 128-130.

Mohsenin, N. N. (1970). Physical Properties of Plant and Animal Materials. London, England: Gordon and Breach Science Publishers.

Paulo, B. B., Ramos, F. de M., \& Prata, A. S. (2017). An investigation of operational parameters of jet cutting method on the size of Ca-alginate beads. Journal of Food Process Engineering 40(6), 1-8.

Prüße, U., Luca, B., Marek, B., Branko, B., Jozef, B., Peter, G., Dorota, L., Verica, M., Benjamin, M., Claudio, N., Viktor, N., Poncelet, D., Swen, S., Lucien, T., Azzurra, T., Alica V., \& Klaus-Dieter V. (2008). Comparison of different technologies for alginate beads production. Chemical Papers 62(4), 364-374.

Prüße, U., Jörg, D., Jürgen, B., \& Klaus-Dieter, V. (2000). Production of spherical beads by JetCutting. Chemical Engineering Technology 23(12), 1105-1110.

Prüße, U., Ulrich, J., Peter, W., Jürgen, B., \& KlausDieter, V. (2002). Bead production with JetCutting and rotating disc/nozzle technologies. Landbauforschung Volkenrode Sonderheft 241, 1-10.
Soliman, E. A., El-Moghazy, A. Y., Mohy El -Din, M. S., \& Massoud, M. A. (2013). Microencapsulation of essential oil within alginate: Formulation and in vitro evaluation of antifungal activity. Journal of Encapsulation and Adsorption Sciences 3, 48-55.

Tan, B., \& Soderstrom, D. N. (1989). Qualitative aspects of UV-vis spectrophotometry of beta-carotene and lycopene. Journal of Chemical Education 66(3), 258.

Truong, V., Nguyen, P. T., Ta, P. N. M., Nguyen, P. T., \& Pham, N. T. C. (2020). Effects of type and concentration of alginate on microencapsulation characteristics of lime essential oil (Citrus aurantifolia) produced by extrusion-dripping methods. The Journal of Agriculture and Development 19(1), 65-76.

Truong, V. (2020). Report of Project B2018-NLS13 funded by Ministry of Education and Training of Vietnam. Ho Chi Minh City, Vietnam.

Xiao, Z., Wanlong, L., Guangyong, Z., Rujun, Z., \& Yunwei, N. (2013). A review of the preparation and application of flavour and essential oils microcapsules based on complex coacervation technology. Journal of the Science of Food and Agricultre 94, 1482-1494. 